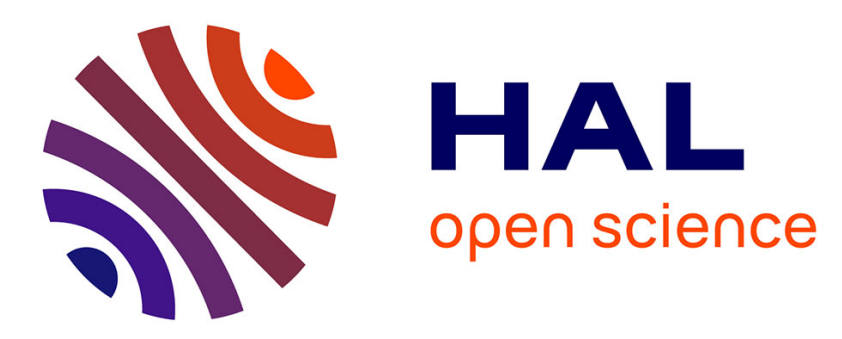

\title{
An evaluation of the sparsity degree for sparse recovery with deterministic measurement matrices
}

Yannick Berthoumieu, Charles H Dossal, Nelly Pustelnik, Philippe Ricoux, Flavius Turcu

\section{- To cite this version:}

Yannick Berthoumieu, Charles H Dossal, Nelly Pustelnik, Philippe Ricoux, Flavius Turcu. An evaluation of the sparsity degree for sparse recovery with deterministic measurement matrices. Journal of Mathematical Imaging and Vision, 2013, 10.1007/s10851-013-0453-4 . hal-00878620

\section{HAL Id: hal-00878620 \\ https://hal.science/hal-00878620}

Submitted on 30 Oct 2013

HAL is a multi-disciplinary open access archive for the deposit and dissemination of scientific research documents, whether they are published or not. The documents may come from teaching and research institutions in France or abroad, or from public or private research centers.
L'archive ouverte pluridisciplinaire HAL, est destinée au dépôt et à la diffusion de documents scientifiques de niveau recherche, publiés ou non, émanant des établissements d'enseignement et de recherche français ou étrangers, des laboratoires publics ou privés. 


\title{
An evaluation of the sparsity degree for sparse recovery with deterministic measurement matrices *
}

\author{
Y. Berthoumieu ${ }^{\dagger}$ \\ Ch. Dossal ${ }^{\ddagger}$ \\ N. Pustelnik ${ }^{\S}$ \\ Ph. Ricoux \\ F. Turcu ${ }^{\dagger}$
}

October 30, 2013

\begin{abstract}
The paper deals with the estimation of the maximal sparsity degree for which a given measurement matrix allows sparse reconstruction through $\ell_{1}$-minimization. This problem is a key issue in different applications featuring particular types of measurement matrices, as for instance in the framework of tomography with low number of views. In this framework, while the exact bound is NP hard to compute, most classical criteria guarantee lower bounds that are numerically too pessimistic. In order to achieve an accurate estimation, we propose an efficient greedy algorithm that provides an upper bound for this maximal sparsity. Based on polytope theory, the algorithm consists in finding sparse vectors that cannot be recovered by $\ell_{1}$-minimization. Moreover, in order to deal with noisy measurements, theoretical conditions leading to a more restrictive but reasonable bounds are investigated. Numerical results are presented for discrete versions of tomography measurement matrices, which are stacked Radon transforms corresponding to different tomograph views.
\end{abstract}

\footnotetext{
${ }^{*}$ Part of this work will appear in the conference proceedings of EUSIPCO 2012 [28]

${ }^{\dagger}$ Y. Berthoumieu and F. Turcu are with Université de Bordeaux, IMS, UMR CNRS 5218, 33405 Talence cedex, France (yannick.berthoumieu@ims-bordeaux.fr, flavius.turcu@ims-bordeaux.fr)

${ }^{\ddagger}$ Ch. Dossal is with Université de Bordeaux, IMB, CNRS UMR 5584, 33405 Talence cedex, France (charles.dossal@math.u-bordeaux1.fr)

${ }^{\S}$ N. Pustelnik is with Laboratoire de Physique de l'ENS Lyon, UMR CNRS 5672, F69007 Lyon, France (nelly.pustelnik@ens-lyon.fr)

${ }^{\top}$ N. Pustelnik is with TOTAL S.A/DG/Direction Scientifique, 92078 PARIS LA DEFENSE Cedex, France (philippe.ricoux@total.com)
} 


\section{Introduction}

The main goal of compressed sensing is the design of sensing matrices $\boldsymbol{A} \in \mathbb{R}^{M \times N}$ with $M \ll N$ for which every $s$-sparse signal $\overline{\boldsymbol{x}} \in \mathbb{R}^{N}$ can be recovered from the observations $\boldsymbol{y}=\boldsymbol{A} \overline{\boldsymbol{x}}+\boldsymbol{n}$ where $\boldsymbol{n} \in \mathbb{R}^{M}$ denotes an additive perturbation [14]. The design consists in specifying a number $M$ of observations as few as possible to ensure the reconstruction of all (or most of) the $s$-sparse signals.

Most results in the literature provide sufficient conditions for the correct reconstruction of signals up to some theoretically guaranteed sparsity, in terms of quantifiable properties of a matrix $\boldsymbol{A}$, such as the coherence [14] or the RIP constants [8]. Since such conditions are not necessary, this guaranteed sparsity is in general lower than the actual maximal sparsity achievable for $\boldsymbol{A}$ (this latter is NP-hard to compute for an arbitrary matrix). The gap between the guaranteed sparsity and the actual sparsity is a challenging issue in practical applications. While it seems to be less significant for some particular classes of matrices, like those having random normalized columns [7, 25], it might get prohibitively large for other types of matrices, such as those issued from tomography applications [27]. In such cases, though the aforementioned criteria give very pessimistic sparsity estimates, correct reconstruction can still be achieved for signals that are significantly less sparse than predicted.

In the paper we tackle this problem through an alternative approach that rather provides upper sparsity bounds instead of lower bounds. More precisely, we propose an algorithm that performs, in the case of an arbitrary matrix $\boldsymbol{A}$, a (relatively) fast search for sparse vectors for which the reconstruction fails. The proposed algorithm is an efficient extension for arbitrary matrices of a greedy algorithm that was given by Dossal et al in [16] in the case of matrices with random normalized columns. The new version of the algorithm is used to numerically study discrete models of tomography sensing matrices, in comparison with standard methods that provide quite pessimistic sparsity bounds. Reconstructing vectors with positive elements is also discussed, as being of interest in the tomography analysis of certain industrial materials having an inherent sparse structure. We also adress the problem of sparsity bounds relatively to the robustness to noise in the reconstruction.

Let us recall the standard framework in compressive sensing. In the noiseless case $(\boldsymbol{n}=0)$, the fact that any $s$-sparse vector $\overline{\boldsymbol{x}}$ can be recovered from the measurement $\boldsymbol{y}=\boldsymbol{A} \overline{\boldsymbol{x}}$ amounts to saying that $\boldsymbol{A}$ is one-to-one when restricted to the set of the $s$-sparse vectors. The latter set can be viewed as the closed ball $B_{0}(s)$ of radius $s$ in $\mathbb{R}^{N}$ under the pseudo-norm

$$
\|\boldsymbol{x}\|_{0}=\#\left\{i: x_{i} \neq 0\right\} \quad \boldsymbol{x}=\left(x_{i}\right)_{1 \leq i \leq N} \in \mathbb{R}^{N} .
$$

This one-to-one behaviour of $\boldsymbol{A}$ means that any $\overline{\boldsymbol{x}}$ in $B_{0}(s)$ is the unique solution of the problem

$$
\underset{\boldsymbol{x} \in \mathbb{R}^{N}}{\operatorname{Argmin}}\|\boldsymbol{x}\|_{0} \quad \text { subject to } \boldsymbol{y}=\boldsymbol{A} \boldsymbol{x} .
$$

In the noisy case $\boldsymbol{n} \neq 0$, the optimization problem is defined as:

$$
\underset{\boldsymbol{x} \in \mathbb{R}^{N}}{\operatorname{Argmin}}\|\boldsymbol{x}\|_{0} \quad \text { subject to } \quad\|\boldsymbol{y}-\boldsymbol{A x}\|_{2} \leq \epsilon,
$$


for suitably chosen $\epsilon>0$. Due to the non-convexity of the problems (1) and (2), solving them by conventional optimization techniques does not guarantee, in general, the convergence to the global minimizer (see a contrario [4]). Moreover, a straightforward approach using combinatorial techniques yields a NP-hard problem. The standard workaround is to convexify (2) by minimizing an $\ell_{1}$-norm instead of the $\ell_{0}$-pseudo-norm, i.e. by considering the optimization problem

$$
\underset{\boldsymbol{x} \in \mathbb{R}^{N}}{\operatorname{Argmin}}\|\boldsymbol{x}\|_{1} \quad \text { subject to } \quad\|\boldsymbol{y}-\boldsymbol{A} \boldsymbol{x}\|_{2} \leq \epsilon .
$$

Unlike $\ell_{0}$-minimization, many tractable algorithms have been proposed to solve problem $(3)$ or its Lagrangian formulation (see $[9,2]$ and references therein), i.e.

$$
\underset{\boldsymbol{x} \in \mathbb{R}^{N}}{\operatorname{Argmin}} \frac{1}{2}\|\boldsymbol{y}-\boldsymbol{A} \boldsymbol{x}\|_{2}^{2}+\gamma\|\boldsymbol{x}\|_{1},
$$

for suitably chosen $\gamma>0$. Since the problems (2) and (3) are not equivalent, a key question is when a sparse vector $\overline{\boldsymbol{x}}$ is the unique solution of the $\ell_{1}$-minimization problem. If this happens, we state that $\overline{\boldsymbol{x}}$ is $\ell_{1}$ - identifiable. In particular, if the $\ell_{1}$ and $\ell_{0}$ problems both have the unique solution $\overline{\boldsymbol{x}}$ for any $\overline{\boldsymbol{x}}$ in $B_{0}(s)$, the matrix $\boldsymbol{A}$ is said to verify the $\ell_{1} / \ell_{0}-$ equivalence up to the sparsity $s$. These properties stand at the core of compressive sensing and the literature offers various techniques to handle this problem $[14,21,13,19,8,33,11]$ :

- A general characterization of the $\ell_{1} / \ell_{0}$ equivalence in the noiseless case is given by Donoho [13] via the notion of neighbourliness from polytope theory; Its dual interpretation is known as the Null Space Property [21, 22].

- Sufficient conditions for $\ell_{1} / \ell_{0}$ equivalence are formulated by Donoho and Huo in terms of matrix coherence [14], and, alternatively, by Candès et al. [8] via the Restricted Isometry Property (RIP);

- Sufficient conditions for $\ell_{1}$-identifiability are given by Fuchs [19] and Tropp [33] using first order necessary conditions and properties of the sub-differential of the $\ell_{1}$ norm.

Such criteria may include robustness to noise [18, 8, 33]. Unfortunately, for a given arbitrary matrix $\boldsymbol{A}$, the main downside is that their numerically evaluation is NP-hard. This is why most results in the literature rather focus on emphasizing classes of matrices that theoretically match such criteria. Typical and widely studied examples are classes of matrices with random normalized columns, which are shown to match RIP-based criteria with high probability, due to their particular eigenvalue distribution [8]. Other examples and interesting proposal can be found in [6], [12], [5], [23], or [24].

Nevertheless, various applications may naturally confine the sensing design to particular classes of matrices that do not fit into these frameworks. This happens for instance in tomography applications, where the sensing matrices consist, within discrete models, in vertically stacked discrete Radon transforms, taken for different polar angles. The design itself is reduced in this context to choosing a suitable set of polar angles. Numerical results suggest that these tomography-related matrices tend to be neither RIP- nor coherence-friendly, in the sense these criteria yield sparsity 
lower-bounds that are too pessimistic. An alternative approach consists in finding upper-bounds instead, which amounts, for a given matrix $\boldsymbol{A}$, to search for unidentifiable vectors as sparse as possible. This strategy was used in the work of Dossal et al [16] in the case of random matrices with normalized columns. The proposed greedy algorithm is related to Donoho's characterization on the $\ell_{1} / \ell_{0}$-equivalence in terms of polytope neighbourliness [13].

In the paper we extend this greedy algorithm in [16] to the case of a general matrix $\boldsymbol{A}$, without a significant complexity increase. We do this in order to numerically study tomography-issued sensing matrices. Since this algorithm covers only the noiseless case, we also propose a way to estimate an approximation of the sparsity degree ensuring $\ell_{1}$-recovery of sparse signals in presence of noise. This latter approach is related to the works of Fuchs [18] and Tropp [33] based on the notion of exact recovery coefficient (ERC).

Notations: Throughout the paper, vectors are denoted by bold lower case letters and matrices by upper case ones. We denote $\left(\boldsymbol{e}_{i}\right)_{1 \leq i \leq N}$ the canonical base in $\mathbb{R}^{N}$. The $i$-th coordinate of a vector $\boldsymbol{x} \in \mathbb{R}^{N}$ is denoted by $x_{i}$ and the $i$-th column of a matrix $\boldsymbol{A} \in \mathbb{R}^{M \times N}$ is denoted by $\boldsymbol{a}_{i}$. We denote by $\boldsymbol{A}_{I} \in \mathbb{R}^{M \times|I|}$ the matrix formed by taking the columns of $\boldsymbol{A}$ indexed by $I \subset\{1, \ldots, N\}$ and by $\boldsymbol{x}_{I}=\left(x_{i}\right)_{i \in I} \in \mathbb{R}^{|I|}$. We define for $p \geq 1$ the $\ell_{p}$-norm of a vector $\boldsymbol{x} \in \mathbb{R}^{N}$ as $\|\boldsymbol{x}\|_{p}=\left(\sum_{i=1}^{N}\left|\boldsymbol{x}_{i}\right|^{p}\right)^{1 / p}$. Finally, the notation $\boldsymbol{A}^{+}$stands for the Moore-Penrose inverse of $A$, and $P_{\boldsymbol{A}}:=\boldsymbol{A} \boldsymbol{A}^{+}$will denote the orthogonal projector onto the range of $\boldsymbol{A}$.

\section{Fast searching non-identifiable vectors for arbitrary matrices.}

In [16], Dossal et al. proposed a greedy algorithm allowing a fast search of $\ell_{1}$-unidentifiable vectors for a sensing matrix $\boldsymbol{A}$ having normalized random columns. In this section an efficient extension of this algorithm is given for an arbitrary sensing matrix $\boldsymbol{A}$. This gives in particular an upper bound for the maximal sparsity for which $\boldsymbol{A}$ still has the $\ell_{1} / \ell_{0}$ - equivalence property.

Let us first recall Donoho's characterization in [13] and the related greedy algorithm in [16]. We denote $B_{1}$ the closed unit ball of $\mathbb{R}^{N}$ relatively to the $\ell_{1}$-norm. The ball $B_{1}$ is a polytope having the signed multiples of the canonical base as vertices, and in which any face is a simplex. Let $P \subset \mathbb{R}^{M}$ be the image of $B_{1}$ under the operator $\boldsymbol{A}$, also called the quotient polytope. Obviously $P$ is the convex hull of the $2 N$ points $\left( \pm \boldsymbol{a}_{i}\right)_{1 \leq i \leq N}$ in $\mathbb{R}^{M}$, which are the images through $\boldsymbol{A}$ of the vertices of $B_{1}$. In general, these points might not be all vertices of $P$, and, similarly, $\boldsymbol{A}$ might not carry faces of $B_{1}$ onto faces of $P$. If however this happens up to an affine dimension say $s$, the polytope $P$ is called $s$-neighbourly. Donoho shows that the $s$-neighbourliness of $P$ is in fact equivalent to the $\ell_{1} / \ell_{0}$ equivalence property of $\boldsymbol{A}$ up to the sparsity $s$.

For a given matrix $\boldsymbol{A}$, the least integer $s$ for which either of the statements above fails is called by Donoho the $\ell_{1} / \ell_{0}$ - equivalence breakdown point (EPB) of $\boldsymbol{A}$. As one can see above, an exact computation of the EBP of an arbitrary matrix is NP-hard, because it involves to examine the images through $\boldsymbol{A}$ of all the faces of $B_{1}$ up to some affine dimension $s$.

However, searching for upper bounds of the EBP is more feasible. According to the above 
characterization, all one needs to do is find, by whatever means, faces of $B_{1}$, of affine dimension as low as possible, that are not sent by $\boldsymbol{A}$ onto faces of $P$, but rather into its convex interior. In order to find such face images, although not rigorously, it is natural to look for them as "inwards" into the polytope $P$ as possible, meaning as close to the origin (the center of $P$ ) as possible.

This is the principle on which the greedy algorithm in [16] is based, and which will be discussed in the following. Let $k \geq 1$ and fix a $(k-1)$ face of $B_{1}$. This can be done by fixing a sign vector $\boldsymbol{\sigma}$ whose support has cardinal $k$ and defining the $(k-1)$-face of $B_{1}$ denoted

$$
F_{\sigma}=\operatorname{Conv}\left\{\sigma_{i} \boldsymbol{e}_{i}: i \in \operatorname{Supp}(\boldsymbol{\sigma})\right\},
$$

which is carried by $\boldsymbol{A}$ onto the subset of $P$ :

$$
\boldsymbol{A} F_{\sigma}=\operatorname{Conv}\left\{\sigma_{i} \boldsymbol{a}_{i}: i \in \operatorname{Supp}(\boldsymbol{\sigma})\right\} .
$$

In order to precise the distance between such a set and the origin in $\mathbb{R}^{M}$ let us consider the following definition. For any $\boldsymbol{x}$ in $\mathbb{R}^{N}$ with support say $I$, let

$$
d(\boldsymbol{x})=\left(\boldsymbol{A}_{I}^{+}\right)^{T} \operatorname{sign}\left(\boldsymbol{x}_{I}\right), \quad \mathcal{D}_{\boldsymbol{x}}=\|d(\boldsymbol{x})\|_{2} .
$$

Then the distance from the origin to $\boldsymbol{A} F_{\boldsymbol{\sigma}}$ is $1 / \mathcal{D}_{\boldsymbol{\sigma}}$. Moreover, the orthogonal affine projection of the origin onto $\boldsymbol{A} F_{\boldsymbol{\sigma}}$ is the vector $d(\boldsymbol{\sigma}) / \mathcal{D}_{\boldsymbol{\sigma}}^{2}$. Note also that, for a given $\boldsymbol{x}$ in $\mathbb{R}^{N}$, both $d(\boldsymbol{x})$ and the $\ell_{1}$-identifiability of $\boldsymbol{x}$ depend only on the sign of $\boldsymbol{x}$. This distance is illustrated in Figure 1.

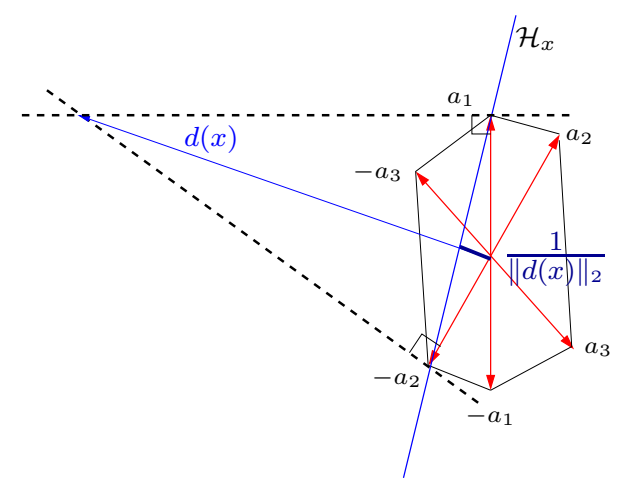

Figure 1: Illustration of $\frac{1}{\|d(\boldsymbol{x})\|_{2}}$ : Distance from the center of the polytope to the hyperplane $\mathcal{H}_{\boldsymbol{x}}$ going through $\left\{\operatorname{sign}\left(\boldsymbol{x}_{i}\right) a_{i}\right\}_{i \in I}$ where $\left.I \subset\{1, \ldots, N\}\right)$.

According to these facts, the greedy algorithm proposed by Dossal et al. [16] looks for sign vectors $\boldsymbol{x}$ that are not $\ell_{1}$-identifiable among those with the largest possible values of $\mathcal{D}_{\boldsymbol{x}}$, i.e. corresponding to faces of $P$ as close to the origin as possible. This algorithm is recalled in Algorithm 1. 


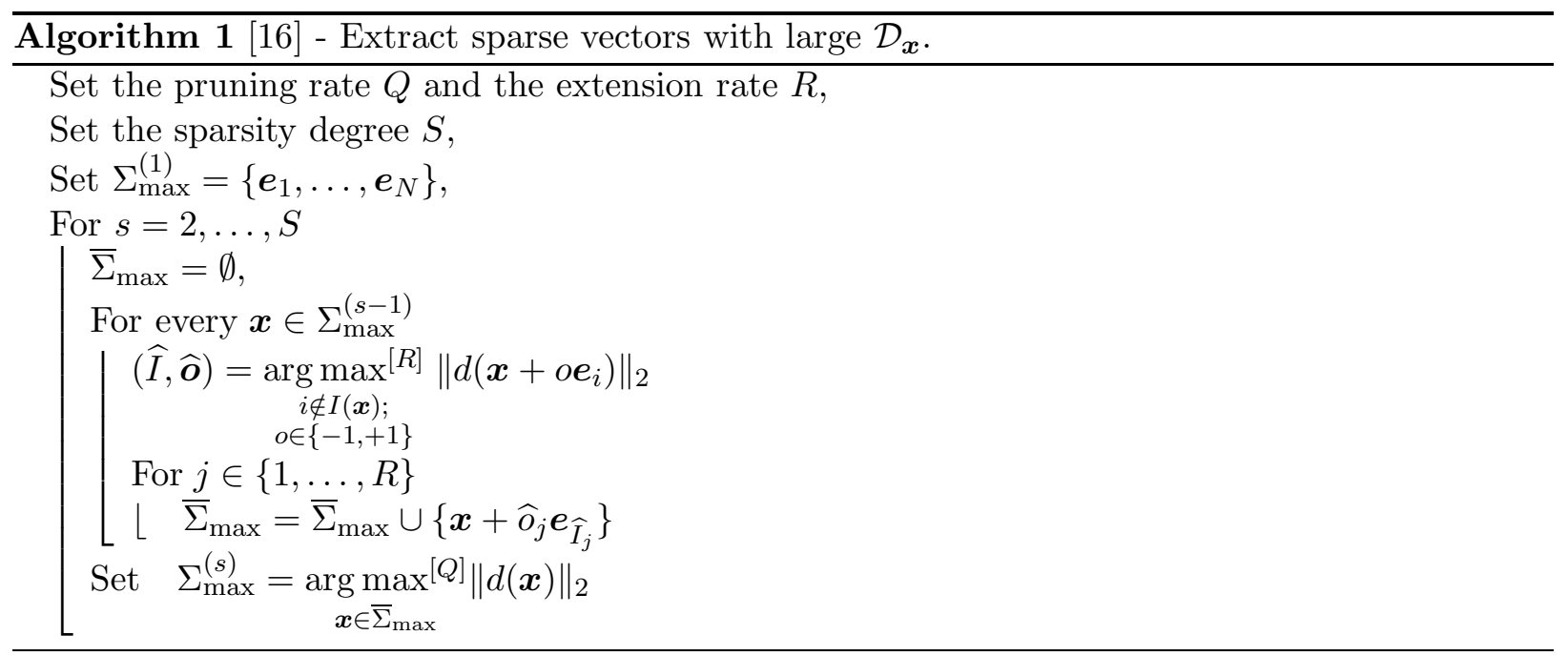

More specifically, each iteration (i.e. for $s=1,2, \ldots$ ) constructs a set, denoted $\Sigma_{\max }^{(s)}$, of $s$-sparse vectors with the largest $\mathcal{D}_{\boldsymbol{x}}$ values, which are candidates to unidentifiability. At each iteration, the new candidate set of $s$-sparse vectors $\Sigma_{\max }^{(s)}$ is built from the previous vector set $\Sigma_{\max }^{(s-1)}$ (e.g. set of vectors with a sparsity degree $s-1)$. It results that each step looks for the $s$-sparse vectors $\widetilde{\boldsymbol{x}}$ such that $\widetilde{\boldsymbol{x}}=\boldsymbol{x}+o \boldsymbol{e}_{i}$ where $\boldsymbol{x}$ denotes a $(s-1)$-sparse vector from $\Sigma_{\max }^{(s-1)}, o \in\{-1,+1\}$ and $\boldsymbol{e}_{i}$ is a Dirac vector at the location $i$. In Algorithm 1 , the notation $\arg \max ^{[R]}$ (resp. $\arg \max { }^{[Q]}$ ) denotes the $R$ pairs $(i, o)$ which correspond to the $R$ largest values of $\left\|d\left(\boldsymbol{x}+o \boldsymbol{e}_{i}\right)\right\|_{2}$ (resp. the $Q$ vectors $\boldsymbol{x}$ yielding the $Q$ largest values of $\left.\|d(\boldsymbol{x})\|_{2}\right)$.

The complexity of Algorithm 1 is given in the following proposition.

Proposition 2.1 For every sparsity degree $s>2$, the complexity of the $s$ step in Algorithm 1 is

$$
O\left(2 Q(N-s+1)\left(N(s+1)+s^{3}\right)\right) \ll O\left(2^{s} \mathrm{C}_{s}^{N}\right) .
$$

Note that the majorant above represents the complexity of the s step in the exact computation of $\operatorname{EBP}(\boldsymbol{A})$.

Proof. Let $\boldsymbol{x}$ belongs to the set $\Sigma_{\max }^{(s-1)}$ which denotes a set of $Q$ vectors of size $s-1$. The computation of $\left\|d\left(\boldsymbol{x}+o \boldsymbol{e}_{i}\right)\right\|_{2}$ has a complexity of $O\left(N+N s+s^{3}\right)$. Moreover, in order to extract the $R$ vectors with the largest $\mathcal{D}_{\boldsymbol{x}}$, the computation of the norm has to be proceeded $2 Q(N-(s-1))$ times. $\mathrm{c}$

Albeit being polynomial, the computational cost of each iteration stays too high to be used in realistic experiments, mainly due to the fourth degree term in $N s^{3}$. However, it is showed in [16] that this can be much improved under the assumption that the matrix $\boldsymbol{A}$ has random normalized columns. Indeed, one can write [16, Proposition 4]:

$$
\|d(\widetilde{\boldsymbol{x}})\|_{2}^{2}=\|d(\boldsymbol{x})\|_{2}^{2}+\left\|\widetilde{\boldsymbol{a}}_{j}\right\|_{2}^{2}\left|\left\langle d(\boldsymbol{x}), \boldsymbol{a}_{j}\right\rangle-o\right|^{2}
$$


where $\boldsymbol{x}$ denotes a $s$-sparse vector with a support $I \subset\{1, \ldots, N\}, o \in\{-1,+1\}, \widetilde{\boldsymbol{x}}$ denotes a $(s+1)$ sparse vector with the support $I \cup\{j\}$ where $j \in\{1, \ldots, N\} \backslash I$, and $\widetilde{\boldsymbol{a}}_{j} \in \operatorname{Span}\left(\boldsymbol{a}_{i}, i \in I \cup\{j\}\right)$ such that $\left\langle\widetilde{\boldsymbol{a}}_{j}, \boldsymbol{a}_{j}\right\rangle=1$, and, for every $i \in I,\left\langle\widetilde{\boldsymbol{a}}_{j}, \boldsymbol{a}_{i}\right\rangle=0$. Under the additional assumption on the columns of $\boldsymbol{A}$, the norms $\left\|\widetilde{\boldsymbol{a}}_{i}\right\|_{2}^{2}$ are close to 1 , so their computation in (6) can be avoided. This leads to an accelerated version of Algorithm 1 for random matrices with normalized columns, which will be refereed as Algorithm 1-bis in the following.

The complexity of this accelerated version of Algorithm 1 is given below.

Proposition 2.2 For every sparsity degree $s>2$, the complexity of Algorithm 1-bis is

$$
O\left(Q\left(N(s-1)+(s-1)^{3}\right)+4 Q N(N-(s-1))\right) .
$$

Proof. Let $\boldsymbol{x}$ belongs to the set $\Sigma_{\max }^{(s-1)}$ which denotes a set of $Q$ vectors of size $s-1$. The computation of $d(\boldsymbol{x})$ has a complexity of $O\left(N(s-1)+(s-1)^{3}\right)$. This computation has to be realized $Q$ times. Moreover, in order to extract the $R$ vectors with the largest $\mathcal{D}_{\boldsymbol{x}}$, the computation of $\|d(\boldsymbol{x})\|_{2}^{2}+\left|\left\langle d(\boldsymbol{x}), \boldsymbol{a}_{i}\right\rangle-o\right|^{2}$, which has a complexity of $O(2 N)$, has to be proceeded $2 Q(N-(s-1))$ times.

In the following we propose an efficient extension of Algorithm 1 in the case of an arbitrary matrix $\boldsymbol{A}$ In this general case, the accelerated version of Algorithm 1 can no longer be used due to the fact that the norms $\left\|\widetilde{\boldsymbol{a}}_{i}\right\|_{2}^{2}$ can no longer be discarded. However, in order to reduce the computational cost of Algorithm 1 (stated in Proposition 2.1), we consider Eq. (6) and derive the closed form of $\left\|\widetilde{\boldsymbol{a}}_{i}\right\|_{2}^{2}$.

Proposition 2.3 Let $j \in\{1, \ldots, N\} \backslash I$ and $\widetilde{\boldsymbol{a}}_{j} \in \mathbb{R}^{M}$ such that

1. $\widetilde{\boldsymbol{a}}_{j} \in \operatorname{Span}\left(\boldsymbol{a}_{i}, i \in I \cup\{j\}\right)$,

2. For every $i \in I,\left\langle\widetilde{\boldsymbol{a}}_{j}, \boldsymbol{a}_{i}\right\rangle=0$,

3. $\left\langle\widetilde{\boldsymbol{a}}_{j}, \boldsymbol{a}_{j}\right\rangle=1$,

then, $\widetilde{\boldsymbol{a}}_{j}$ can be expressed as follows:

$$
\widetilde{\boldsymbol{a}}_{j}=\frac{\boldsymbol{a}_{j}-P_{\boldsymbol{A}_{I}} \boldsymbol{a}_{j}}{\left\langle\boldsymbol{a}_{j}, \boldsymbol{a}_{j}-P_{\boldsymbol{A}_{I}} \boldsymbol{a}_{j}\right\rangle}
$$

Proof. For every $\widetilde{\boldsymbol{a}}_{j} \in \mathbb{R}^{M}$, it exists $\kappa \in \mathbb{R}$ and $\boldsymbol{a} \in \mathbb{R}^{M}$ such that $\widetilde{\boldsymbol{a}}_{j}=\kappa \boldsymbol{a}$. Assumptions (i) and (ii) yield to

$$
\boldsymbol{a}=\boldsymbol{a}_{j}-P_{\boldsymbol{A}_{I}} \boldsymbol{a}_{j}
$$

From Assumption 3., we can write $\left\langle\kappa \boldsymbol{a}, \boldsymbol{a}_{j}\right\rangle=1$, and then

$$
\kappa=\frac{1}{\left\langle\boldsymbol{a}, \boldsymbol{a}_{j}\right\rangle} .
$$


The combination of (8) and (9) leads to (7).

The computation of $d(\widetilde{\boldsymbol{x}})$ can thus be expressed as a function of $d(\boldsymbol{x})$ and $\widetilde{\boldsymbol{a}}_{j}$. For each sparsity degree $s$ in Algorithm 1, this expression leads to the computation of $Q$ matrix inversions of size $(s-1) \times(s-1)$ rather than $Q \times(N-s)$ matrix inversions of size $s \times s$. The proposed algorithm is detailed in Algorithm 2 and the associated computational cost is specified in Proposition 2.4.

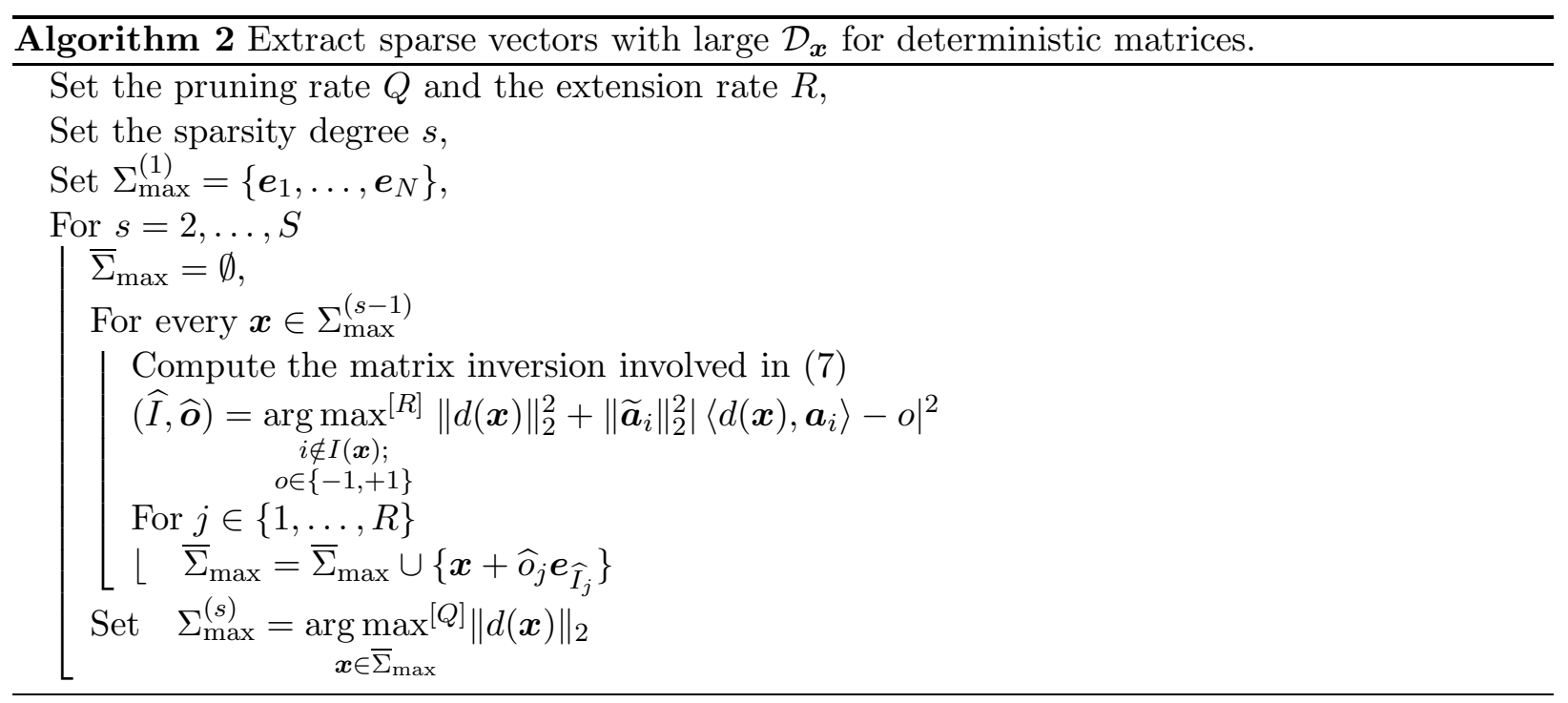

Proposition 2.4 For every sparsity degree $s>2$, the complexity of the $s$-th step in Algorithm 2 is

$$
O\left(Q\left(N(s-1)+(s-1)^{3}\right)+2 Q(N-(s-1))(N(s+4))\right) .
$$

Proof. Let $\boldsymbol{x}$ belongs to the set $\Sigma_{\max }^{(s-1)}$ which denotes a set of $Q$ vectors of size $k-1$. The complexity of $\boldsymbol{A}_{I}\left(\boldsymbol{A}_{I}^{*} \boldsymbol{A}_{I}\right)^{-1}$ has a complexity of $O\left(N(s-1)+(s-1)^{3}\right)$. This computation has to be done $Q$ times. Moreover, in order to extract the $R$ vectors with the largest $\mathcal{D}_{\boldsymbol{x}}$, the computation of $\|d(\boldsymbol{x})\|_{2}^{2}+\left\|\tilde{\boldsymbol{a}}_{i}\right\|_{2}^{2}\left|\left\langle d(\boldsymbol{x}), \boldsymbol{a}_{i}\right\rangle-o\right|^{2}$ requires $2 N+N(s+2)$ operations which are proceeded $2 Q(N-$ $(s-1))$ times.

Note that this complexity is significantly lower than in Algorithm 1, as it no longer has the fourth degree term.

Remark 2.5 Note that the "minimum version of Algorithm 2" is presented in Algorithm 3. This algorithm makes it possible to extract sparse vectors with small $\mathcal{D}_{\boldsymbol{x}}$, which leads to $\ell_{1}$-identifiable vectors. The complexity of each iteration is similar to Algorithm 2.

Remark 2.6 In practice the inverse of $\boldsymbol{A}_{I}^{*} \boldsymbol{A}_{I}$ can be computed in different ways, with timecomputational cost less than $s^{3}$ as the sparsity $s$ increases. To do so, one could use for instance the Schur decomposition to compute inverses at the $s$-th step from the inverse already computed at the step $s-1$. However, such a solution would require to keep in memory $Q$ matrices $\left(A_{I}^{*} A_{I}\right)^{-1}$ 


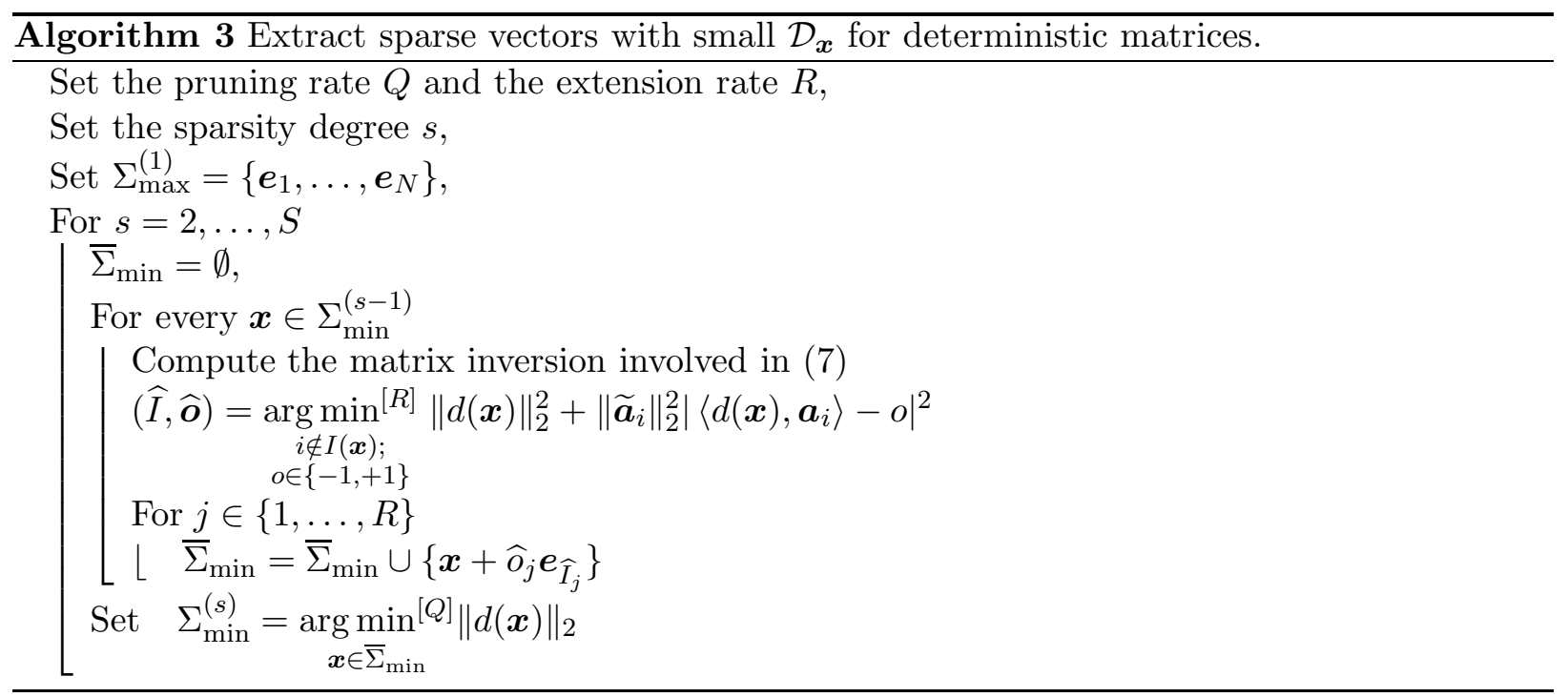

of size $s$ at each step $s$ of the algorithm. Since such trade-offs may or not be beneficial for various implementations, we evaluate, as a reference, the computational cost for Algorithms 1, 1-bis and 2 with the $s^{3}$ term, corresponding to a standard (non-iterative) inversion for each step $s$.

Remark 2.7 In Figure 2 we highlight the behaviour of the complexity according to different sparsity regimes. The complexity of Algorithm 1 is plotted in black, the complexity for the accelerated version in the random case in represented by the blue line and the red one denotes the complexity of proposed algorithm i.e. Algorithm 2. It clearly appears that for small regime of sparsity i.e. $s<<N$ Algorithm 1 and 2 have a similar behaviour while for s greater than $\sqrt{N}$ Algorithm 2 outperforms Algorithm 1. The same behaviour is observed for different values of $N$. In this figure $N=2500$.

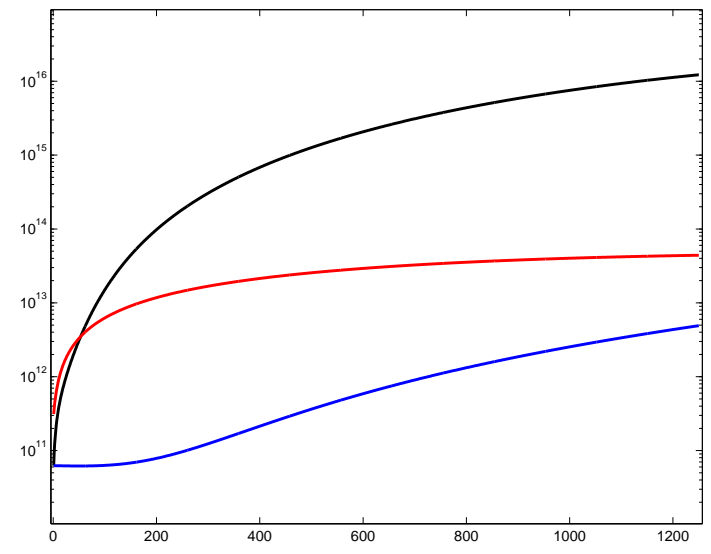

Figure 2: Graphical comparison of the complexities as a fonction of the sparsity when $N=2500$. The complexity of Algorithm 1 is plotted in black, the complexity for the accelerated version in the random case in represented by the blue line and the red one denotes the complexity of the proposed algorithm i.e. Algorithm 2. 
Algorithm 2 allows us to extract the $s$-sparse vectors with the largest value of $\mathcal{D}_{\boldsymbol{x}}$. It results that if the vector $\boldsymbol{x} \in \Sigma_{\max }^{(s)}$ having the largest $\mathcal{D}_{\boldsymbol{x}}$ value cannot be recovered by $\ell_{1}$-minimization, a fair estimation of the sparsity degree $s$ for which every $s$-sparse vectors can be reconstructed by $\ell_{1}$-minimization from $\boldsymbol{y}=\boldsymbol{A} \overline{\boldsymbol{x}}$ is the largest $s^{\prime}<s$. The algorithm to extract this approximated sparsity is summed up by Algorithm 4.

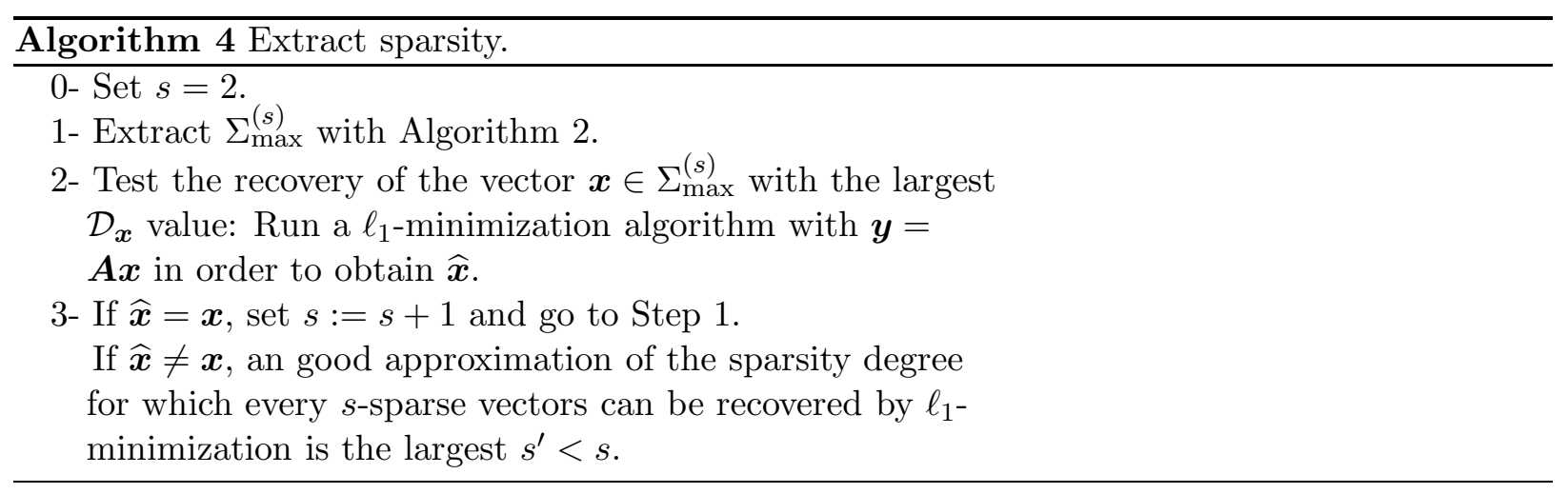

\section{Noisy case}

As mentioned in the introduction, using first order necessary condition and the sub-differential of the $\ell_{1}$ norm, Fuchs [19] and Tropp [33] derived sparse $\ell_{1}$-recovery conditions in the noisy case. In this section we extend their results in order to control the reconstruction error in the noisy case. The first result provides guarantees on the support of the solution while the second one does not have this support guaranty, but it takes into account the sign of the solution, which may allow to relax the maximal sparsity upper bound.

The arguments in the proofs of these results are similar to the ideas raised in [33], but since the context here is slightly different we give the detailed proofs for sake of completeness.

Proposition 3.1 Let $I \subset\{1, \ldots, N\},|I|=s$, such that $\boldsymbol{A}_{I}$ is full rank, and let $\boldsymbol{y}=\boldsymbol{A} \overline{\boldsymbol{x}}+\boldsymbol{n}$ for some vector $\overline{\boldsymbol{x}}$ with support $I$. Denote $J=\{1, \ldots, N\} \backslash I$,

$$
\operatorname{ERC}(I)=\max _{j \in J}\left\|\left(\boldsymbol{A}_{I}^{*} \boldsymbol{A}_{I}\right)^{-1} \boldsymbol{A}_{I}^{*} \boldsymbol{a}_{j}\right\|_{1},
$$

and suppose that:

(i) $\operatorname{ERC}(I)<1$,

(ii) for every $\varepsilon>0$ such that $\gamma=\frac{\max _{j \in J}\left\|\boldsymbol{a}_{j}\right\|_{2}}{1-\operatorname{ERC}(I)} \varepsilon$ and $\|\boldsymbol{n}\|_{2}<\varepsilon$.

Then the $\ell_{1}$ minimization problem (4) has a unique solution $\widehat{\boldsymbol{x}}$ with the following properties:

1. the support of $\widehat{\boldsymbol{x}}$ is included in the support of $\overline{\boldsymbol{x}}$, 
2. the error resulting from the noise verifies:

$$
\|\widehat{\boldsymbol{x}}-\overline{\boldsymbol{x}}\|_{2} \leq\left(\lambda_{\min }\left(\boldsymbol{A}_{I}^{*} \boldsymbol{A}_{I}\right)\right)^{-1}\left(\left\|\boldsymbol{A}_{I}^{*} \boldsymbol{n}\right\|_{2}+\frac{\sqrt{|I|} \max _{j \in J}\left\|\boldsymbol{a}_{j}\right\|_{2}}{1-\operatorname{ERC}(I)} \varepsilon\right) .
$$

Proof. We first consider the problem:

$$
\widehat{\boldsymbol{u}}=\underset{\boldsymbol{u} \in \mathbb{R}^{|I|}}{\arg \min } \frac{1}{2}\left\|\boldsymbol{y}-\boldsymbol{A}_{I} \boldsymbol{u}\right\|_{2}^{2}+\gamma\|\boldsymbol{u}\|_{1},
$$

and let $\widetilde{\boldsymbol{u}} \in \mathbb{R}^{K}$ be the extension of the vector $\widehat{\boldsymbol{u}}$ in $\mathbb{R}^{N}$ by adding zeros at the locations $J=$ $\{1, \ldots, N\} \backslash I$.

Considering assumptions (i) and (ii), we want to prove that $\widetilde{\boldsymbol{u}}=\widehat{\boldsymbol{x}}$. Let $I_{0} \subset I$ denote the non-zero components of $\widetilde{\boldsymbol{u}}$ and $J_{0}=\{1, \ldots, N\} \backslash I_{0}$. According to Eq. (11), we have

$$
\boldsymbol{A}_{I_{0}}^{*}\left(\boldsymbol{y}-\boldsymbol{A}_{I} \widehat{\boldsymbol{u}}\right)=\gamma \operatorname{sign}\left(\widehat{\boldsymbol{u}}_{I_{0}}\right),
$$

and

$$
\left(\forall i \in I \cap J_{0}\right), \quad\left|\boldsymbol{a}_{i}^{*}\left(\boldsymbol{y}-\boldsymbol{A}_{I} \widehat{\boldsymbol{u}}\right)\right| \leq \gamma .
$$

The combination of Eqs. (12) and (13) leads to

$$
\begin{aligned}
\boldsymbol{A}_{I_{0}}^{*}(\boldsymbol{y}-\boldsymbol{A} \widetilde{\boldsymbol{u}}) & =\boldsymbol{A}_{I_{0}}^{*}\left(\boldsymbol{y}-\boldsymbol{A}_{I} \widehat{\boldsymbol{u}}\right) \\
& =\gamma \operatorname{sign}\left(\widehat{\boldsymbol{u}}_{I_{0}}\right), \\
& =\gamma \operatorname{sign}\left(\widetilde{\boldsymbol{u}}_{I_{0}}\right),
\end{aligned}
$$

and, for every $i \in I \cap J_{0}$,

$$
\left|\boldsymbol{a}_{i}^{*}(\boldsymbol{y}-\boldsymbol{A} \widetilde{\boldsymbol{u}})\right|=\left|\boldsymbol{a}_{i}^{*}\left(\boldsymbol{y}-\boldsymbol{A}_{I} \widehat{\boldsymbol{u}}\right)\right| \leq \gamma
$$

Let us also note that

$$
\left|\boldsymbol{a}_{j}^{*}\left(P_{\boldsymbol{A}_{I}}(\boldsymbol{y})-\boldsymbol{A}_{I} \widehat{\boldsymbol{u}}\right)\right| \leq \gamma \operatorname{ERC}(I) .
$$

In fact, for any $\boldsymbol{z}$ in the range of $\boldsymbol{A}_{I}$ one has

$$
\begin{aligned}
\max _{j \notin I}\left|\boldsymbol{a}_{j}^{*} \boldsymbol{z}\right| & =\max _{j \notin I}\left|\boldsymbol{a}_{j}^{*} \boldsymbol{A}_{I}\left(\boldsymbol{A}_{I}^{*} \boldsymbol{A}_{I}\right)^{-1} \boldsymbol{A}_{I}^{*} \boldsymbol{z}\right| \\
& \leq \max _{j \notin I}\left\|\boldsymbol{a}_{j}^{*} \boldsymbol{A}_{I}\left(\boldsymbol{A}_{I}^{*} \boldsymbol{A}_{I}\right)^{-1}\right\|_{1}\left\|\boldsymbol{A}_{I}^{*} \boldsymbol{z}\right\|_{\infty} \\
& =\operatorname{ERC}(I)\left\|\boldsymbol{A}_{I}^{*} \boldsymbol{z}\right\|_{\infty}
\end{aligned}
$$

This immediately implies (16) by taking $\boldsymbol{z}=P_{\boldsymbol{A}_{I}}(\boldsymbol{y})-\boldsymbol{A}_{I} \widehat{\boldsymbol{u}}$ and using (14).

Now, for every $j \in J$,

$$
\begin{aligned}
\left|\boldsymbol{a}_{j}^{*}(\boldsymbol{y}-\boldsymbol{A} \widetilde{\boldsymbol{u}})\right| & =\left|\boldsymbol{a}_{j}^{*}\left(\boldsymbol{y}-\boldsymbol{A}_{I} \widehat{\boldsymbol{u}}\right)\right| \\
& =\left|\boldsymbol{a}_{j}^{*}\left(P_{\boldsymbol{A}_{I}}(\boldsymbol{y})+P_{\boldsymbol{A}_{I}}(\boldsymbol{y})-\boldsymbol{A}_{I} \widehat{\boldsymbol{u}}\right)\right| \\
& \leq\left|\boldsymbol{a}_{j}^{*} P_{\boldsymbol{A}_{I}}(\boldsymbol{n})\right|+\left|\boldsymbol{a}_{j}^{*}\left(P_{\boldsymbol{A}_{I}}(\boldsymbol{y})-\boldsymbol{A}_{I} \widehat{\boldsymbol{u}}\right)\right| \\
& \leq\left|\boldsymbol{a}_{j}^{*} P_{\boldsymbol{A}_{I}}(\boldsymbol{n})\right|+\gamma \operatorname{ERC}(I) \\
& \leq\left\|\boldsymbol{a}_{j}\right\|_{2}\left\|P_{\boldsymbol{A}_{I}}(\boldsymbol{n})\right\|_{2}+\gamma \operatorname{ERC}(I) \\
& \leq \varepsilon \max _{j \in J}\left\|\boldsymbol{a}_{j}\right\|_{2}+\gamma \operatorname{ERC}(I)
\end{aligned}
$$


Eq. (18) and assumption (ii) yield, for every $j \in J$,

$$
\left|\boldsymbol{a}_{j}^{*}(\boldsymbol{y}-\boldsymbol{A} \widetilde{\boldsymbol{u}})\right| \leq \gamma
$$

and from Eqs. (13), (14), (19), and $\boldsymbol{A}_{I}$ injectivity, we can conclude that $\widetilde{\boldsymbol{u}}=\widehat{\boldsymbol{x}}$.

To check that $\widetilde{\boldsymbol{u}}$ is unique, suppose $\widetilde{\boldsymbol{v}}$ is another solution of the $\ell_{1}$-minimization minimization problem (4). Then $\boldsymbol{y}=\boldsymbol{A} \widetilde{\boldsymbol{u}}=\boldsymbol{A} \widetilde{\boldsymbol{v}}$ and, moreover, both $\widetilde{\boldsymbol{u}}$ and $\widetilde{\boldsymbol{v}}$ are supported on $I$. But there $\boldsymbol{A}$ coincides with $\boldsymbol{A}_{\boldsymbol{I}}$, which is full rank, thus $\widetilde{\boldsymbol{u}}$ and $\widetilde{\boldsymbol{v}}$ necessarily coincide.

The last part of this proof concerns the control of the error in $\ell_{2}$-norm. Let $\widetilde{\boldsymbol{y}}$ denote the orthogonal projection of $\boldsymbol{y}$ onto the range of $\boldsymbol{A}_{I}$,

$$
\widetilde{\boldsymbol{y}}=P_{\boldsymbol{A}_{I}} \boldsymbol{y}
$$

and let

$$
\begin{aligned}
\widetilde{\boldsymbol{x}} & =\left(\boldsymbol{A}_{I}^{*} \boldsymbol{A}_{I}\right)^{-1} \boldsymbol{A}_{I}^{*} \boldsymbol{y} \\
& =\overline{\boldsymbol{x}}_{I}+\left(\boldsymbol{A}_{I}^{*} \boldsymbol{A}_{I}\right)^{-1} \boldsymbol{A}_{I}^{*} \boldsymbol{n} .
\end{aligned}
$$

Since $\boldsymbol{A}_{I}$ is full rank, $\widetilde{\boldsymbol{x}}$ is the unique vector whose image by $\boldsymbol{A}_{I}$ is $\widetilde{\boldsymbol{y}}$. Locally, the solution of the minimization problem (11) is such that:

$$
\widehat{\boldsymbol{u}}=\left(\boldsymbol{A}_{I}^{*} \boldsymbol{A}_{I}\right)^{-1} \boldsymbol{A}_{I}^{*} \boldsymbol{y}-\gamma\left(\boldsymbol{A}_{I}^{*} \boldsymbol{A}_{I}\right)^{-1} \boldsymbol{s}
$$

where $\boldsymbol{s}=\left(\operatorname{sign}\left(\widetilde{\boldsymbol{u}}_{i}\right)\right)_{i \in I}$. Regarding Eq. (22), we can write

$$
\begin{aligned}
\|\widetilde{\boldsymbol{x}}-\widehat{\boldsymbol{u}}\|_{2} & \left.\leq \gamma \max _{\boldsymbol{s}, I} \|\left(\boldsymbol{A}_{I}^{*} \boldsymbol{A}_{I}\right)\right)^{-1} \boldsymbol{s} \| \\
& \leq \gamma \sqrt{|I|}\left(\lambda_{\min }\left(\boldsymbol{A}_{I}^{*} \boldsymbol{A}_{I}\right)\right)^{-1} .
\end{aligned}
$$

Finally,

$$
\begin{aligned}
\|\overline{\boldsymbol{x}}-\widehat{\boldsymbol{x}}\|_{2} & =\|\overline{\boldsymbol{x}}-\widetilde{\boldsymbol{u}}\|_{2} \\
& =\left\|\overline{\boldsymbol{x}}_{I}-\widehat{\boldsymbol{u}}\right\|_{2} \\
& \leq\left\|\overline{\boldsymbol{x}}_{I}-\widetilde{\boldsymbol{x}}\right\|_{2}+\|\widetilde{\boldsymbol{x}}-\widehat{\boldsymbol{u}}\|_{2} \\
& \leq\left\|\left(\boldsymbol{A}_{I}^{*} \boldsymbol{A}_{I}\right)^{-1} \boldsymbol{A}_{I}^{*} \boldsymbol{n}\right\|_{2}+\gamma \sqrt{|I|}\left(\lambda_{\min }\left(\boldsymbol{A}_{I}^{*} \boldsymbol{A}_{I}\right)\right)^{-1}
\end{aligned}
$$

Considering assumption (ii) and taking into account that

$$
\left\|\left(\boldsymbol{A}_{I}^{*} \boldsymbol{A}_{I}\right)^{-1} \boldsymbol{A}_{I}^{*} \boldsymbol{n}\right\|_{2} \leq\left(\lambda_{\min }\left(\boldsymbol{A}_{I}^{*} \boldsymbol{A}_{I}\right)\right)^{-1}\left\|\boldsymbol{A}_{I}^{*} \boldsymbol{n}\right\|_{2}
$$

the proof is complete.

The second result gives also a bound of the error between the objective vector $\overline{\boldsymbol{x}}$ and the solution of (2), under a weaker assumption than ERC $<1$. 
Proposition 3.2 Let $I \subset\{1, \ldots, N\},|I|=s$, such that $\boldsymbol{A}_{I}$ is full rank, $\boldsymbol{y}=\boldsymbol{A} \overline{\boldsymbol{x}}+\boldsymbol{n}$ for some $\overline{\boldsymbol{x}}$ with support $I$ and let $J=\{1, \ldots, N\} \backslash I$. If, for every $j \in J,\left|\boldsymbol{a}_{j}^{*} d(\overline{\boldsymbol{x}})\right|<1$, with $d(\overline{\boldsymbol{x}})=$ $\boldsymbol{A}_{I}\left(\boldsymbol{A}_{I}^{*} \boldsymbol{A}_{I}\right)^{-1} \operatorname{sign}\left(\overline{\boldsymbol{x}}_{I}\right)$, then, all the solution $\widehat{\boldsymbol{x}}$ of the $\ell_{1}$-minimization problem (4) satisfy

$$
\begin{aligned}
\|\widehat{\boldsymbol{x}}-\overline{\boldsymbol{x}}\|_{2} \leq\left(\|\boldsymbol{n}\|_{2}+\frac{\gamma}{2}\|d(\overline{\boldsymbol{x}})\|_{2}\right)\left(2\left\|\left(\boldsymbol{A}_{I}^{*} \boldsymbol{A}_{I}\right)^{-1} \boldsymbol{A}_{I}^{*}\right\|_{2}\right. \\
\\
\left.+\frac{1+\left\|\left(\boldsymbol{A}_{I}^{*} \boldsymbol{A}_{I}\right)^{-1} \boldsymbol{A}_{I}^{*}\right\|_{2}\left\|\boldsymbol{A}_{J}\right\|_{2}}{1-\max _{j \in J}\left|\boldsymbol{a}_{j}^{*} d(\overline{\boldsymbol{x}})\right|}\left(\frac{\|\|_{2}}{\gamma}+\frac{\|d(\overline{\boldsymbol{x}})\|_{2}}{2}\right)\right)
\end{aligned}
$$

Proof. It follows [20, Proposition 3.13] with the dual certificate $\eta=d(\overline{\boldsymbol{x}}), \delta=\|\boldsymbol{n}\|_{2}$, and $C=\frac{\gamma}{\|\boldsymbol{n}\|_{2}}$.

$\mathrm{Q}$

Let us remark that, in contrast to Proposition 3.1, the sign of $\overline{\boldsymbol{x}}$ appears in the hypothesis of the latter proposition, which makes it a weaker requirement than ERC $<1$. This means that the maximal sparsity for which the condition in Proposition 3.2 holds is higher than the maximal sparsity required so that the exact recovery coefficient be less than 1 . As such, whenever one looks only for positive solutions $\overline{\boldsymbol{x}}$ (as in tomography applications where data are positive) the two propositions give similar sparsity bounds.

One can also remark that Proposition 3.2 does not require an assumption on $\gamma$ contrary to Proposition 3.1. The consequence of this is twofold: first, the minimizer in Proposition 3.2 is not unique, unlike Proposition 3.1, and second, there is no guarantee on partial support recovery in Proposition 3.2, while this is true for Proposition 3.1.

Moreover, one can note that Proposition 3.2 gives a bound on the distance of a point $\bar{x}$ to the set of minimizers, which is non-empty.

\section{Experimental results for tomography-like experiments}

In this part, we evaluate the theoretical results we have proposed in the tomography context. The goal consists to reconstruct an image from its line integral measurements i.e. views. One would like to reconstruct a high-resolution image with a minimal set of measured data. This question was already addressed in a seminal work by Cormack: "In practice one can make only a finite number of measurements with beams of finite width, and the question which arises is how many observations should be made, and how should they be related to each other in order to reconstruct the object" [10]. Cormack explored geometric arguments while further works used two-dimensional sampling theory [26, 29, 17]. Here, we address this question from a compressed sensing point of view.

We denote $\theta=(\cos \varphi, \sin \varphi)$ the unit vector in $\mathbb{R}^{2}$ with polar angle $\varphi$ and $\theta^{\perp}=(\cos \varphi,-\sin \varphi)$. We assume that the image to be reconstructed $\mathrm{x} \in C_{0}^{\infty}(\Omega)$, i.e. $\mathrm{x}$ is infinitely differentiable and vanishes outside the unit disk $\Omega$ of $\mathbb{R}^{2}$. The 2-D Radon transform maps a density function $\mathrm{x}$ into 
its line integral such that:

$$
\mathcal{R} \times(\varphi, s)=\int_{\mathbb{R}} \times\left(s \theta+t \theta^{\perp}\right) d t .
$$

The goal of X-ray tomography is to reconsruct an approximation of $\mathrm{x}$ from sampled values of $\mathcal{R} \mathrm{x}$.

In the following, the sensing matrix $\boldsymbol{A}$ consists in vertically stacked discrete Radon transforms with the MATLAB ${ }^{\complement}$ implementation. The size $N$ of $\boldsymbol{A}$ is the size of the discrete data measured. The number $M$ of lines of $\boldsymbol{A}$ is the product between the number of polar angles (views) and the number of beams in the discrete Radon transform, taken at about twice the data's resolution.

As stated in the previous sections, we simplify the real noise degradation model, which is multiplicative, by an additive noise in $\boldsymbol{y}=\boldsymbol{A} \overline{\boldsymbol{x}}+\boldsymbol{n}$. From a variational point of view, taking into account Poisson multiplicative noise may lead to replace the quadratic data fidelity term in (2)-(4) with a Kullback-Leibler divergence. This can be explained through a MAP formulation where the likelihood models Poisson distribution. However for a low noise level (small value of $\sigma)$, the improvement obtained by considering the Poisson antilog-likelihood rather than $\ell_{2}$ norm is not clear [31]. In our experiments we focus on cases where the noise has a small variance. Dealing with $\ell_{2}$-norm rather than Kullback-Leibler divergence does not appear unrealistic.

The numerical results are organized as follows: (i) we first compare the complexity of the proposed algorithm to the state-of-the-art algorithms through simulated data, (ii) we compare the sparsity bound estimated by the proposed algorithm to the sparsity lower bounds given by classical methods, based on coherence and ERC, (iii) we focus on the performances of a uniform sampling versus a random sampling for a tomography measurement matrix, defined from a uniform or random selection of the polar angles, and (iv) we finally use the bound in Proposition 5 in order to estimate an upper sparsity bound for $\ell_{1}$-recovery in the noisy case.

\subsection{Evaluation of the greedy algorithms}

First, the performance of the proposed greedy algorithm in terms of computational complexity and of maximum extracted $\mathcal{D}_{\boldsymbol{x}}$ values is evaluated.

We compare the original algorithm (Algorithm 1), the accelerated version of previous one designed for random matrices (Algorithm 1-bis), and the proposed fast version devoted to the deterministic matrices (Algorithm 2). The evaluation is carried out both in a context of tomography and of random matrices, i.e. $\boldsymbol{A}$ denotes either a Radon transform where $N=20 \times 20$ and $M=198$ (that corresponds to 4 angles) or a random matrix with the same size. The pruning rate $Q$ and the extension rate $R$ are fixed to $Q=N$ and $R=1$. The results are presented in Figure 3 . 

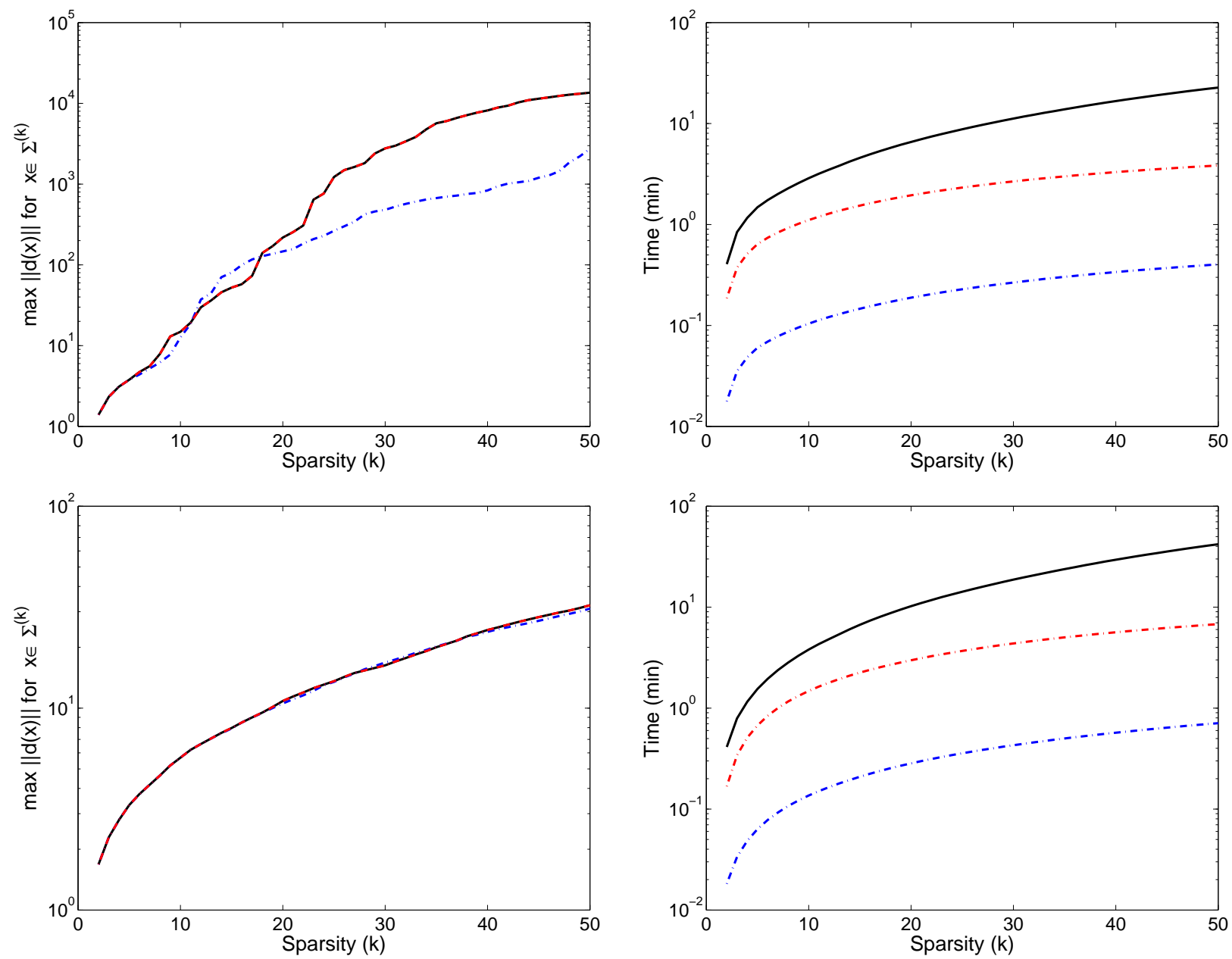

Figure 3: Algorihm 1 (solid black), Algorithm 1-bis (dash-dotted blue), Algorithm 2 (dash-dotted red). The top figures present the results obtained with a tomography-like matrix while the bottom figures illustrate the results for a normalized random matrix. On the left, the figures give the performance in term of maximum extracted $\mathcal{D}_{\boldsymbol{x}}$ values while the right figures present the performance in term of computational time.

One can observe that in a deterministic context (top figures), the extraction performance i.e. find sparse vectors with large $\mathcal{D}_{\boldsymbol{x}}$ (left figure) of Algorithm 2 are similar to those of Algorithm 1 with a much better convergence rate (right figure) while the extraction performance is better than the accelerated version considering Algorithm 1-bis. The saving computational time can be counted up in minutes. However, note that in a random context (bottom figures), the proposed approach leads to smaller improvement. To sum up, the obtained results illustrate the relevance of the proposed algorithm in order to easily handle deterministic matrices with higher dimensionality. 


\subsection{Use Algorithm 2 in order to deal with noisy data and positivity}

It appears that some materials which requires to be studied through a tomographic process exhibit sparse properties, which means that the vector $\boldsymbol{x}$ is positive. The goal of this experiment is to design the measurement matrix $\boldsymbol{A}$ i.e. find the adapted number of views according to a given sparsity degree. Due to the positivity of the data, we proceed Algorithm 2 with $o=+1$.

The experiments have been held for images of size $N=32 \times 32$. We evaluate the performance for different designs of the sensing matrix. For instance, we consider the case where the angle between two views is fixed but also when the angle are randomly selected. Moreover, the case of 2 views $(M=98), 4$ views $(M=196)$, and 6 views $(M=294)$ are studied. The pruning rate $Q$ and the extension rate $R$ are fixed to $Q=N$ and $R=1$.

In these experiments the $\ell_{1}$-minimization algorithm is FISTA [3] and the stopping criterion takes in consideration the evolution of the relative error between $\overline{\boldsymbol{x}}$ and $\widehat{\boldsymbol{x}}\left(<10^{-3}\right)$ as well as the iteration number $\left(\leq 10^{4}\right)$.

Comparison with the state-of-the-art methods - In Table 1, we compare the sparsity degree extracted with the proposed method i.e. Algorithm 4 and the sparsity obtained with the state of the art methods (coherence and ERC) in the noiseless case.

The first row presents the sparsity obtained with the coherence method [19], computed as in [21, Theorem 1]. The second row gives the sparsity obtained in order to have $\operatorname{ERC}(I)<1$ (cf. Proposition 3.1). Note that $\operatorname{ERC}(I)$ is NP-hard to compute but here we consider the support $I$ associated to $\boldsymbol{x} \in \Sigma_{\max }^{(s)}$ with the largest $\mathcal{D}_{x}$ value, which is extracted with Algorithm 2. The last row presents the sparsity degree extracted with the proposed approach described in Algorithm 4. Note that the results obtained with RIP are not presented here because it leads to a sparsity smaller than 1.

The proposed method allows us to reach higher sparsity degree. From this table, it is possible to know the best design of $\boldsymbol{A}$ according to a given sparsity degree. For instance, according to a sparsity of 15 , the best design for the tomographic matrix involves 4 views.

Random versus uniform measurements - The randomness has been often addressed in the literature of compressed sensing for tomography [7, 25, 27]. Consequently we propose to evaluate the impact of the tomography sampling, i.e. uniformly or randomly chosen angles of view, on the sparsity extracted with Algorithm 4.

In Table 2, the first row gives the sparsity in a context of a uniform sampling. The tenth following rows present the sparsity extracted with Algorithm 4 when the angles are randomly selected. The last three rows present the minimum, maximum, and mean sparsity according to the random sampling results.

It appears that the difference in term of extracted sparsity between a random selection of the polar angles and a uniform one is not significant. According to the work by Arias-Castro et al. 


\begin{tabular}{|c|c|c|c|}
\hline & $\begin{array}{c}2 \text { views } \\
M=98\end{array}$ & $\begin{array}{c}4 \text { views } \\
M=196\end{array}$ & $\begin{array}{c}6 \text { views } \\
M=294\end{array}$ \\
\hline \hline $\begin{array}{c}\text { Sparsity } \\
\text { (Coherence) }\end{array}$ & 1 & 1 & 2 \\
\hline $\begin{array}{c}\text { Sparsity } \\
(\text { ERC }<1)\end{array}$ & 4 & 10 & 11 \\
\hline $\begin{array}{c}\text { Sparsity } \\
\text { (Proposed method) }\end{array}$ & $\mathbf{1 0}$ & $\mathbf{3 2}$ & $\mathbf{5 0}$ \\
\hline
\end{tabular}

Table 1: Sparsity $s$ allowing us to recover every $s$-sparse vectors by $\ell_{1}$-minimization in the absence of noise consideration. The last row presents the sparsity extracted with Algorithm 4. The results are given for three different configurations of the tomography-like matrix $\boldsymbol{A}$.

[1], there exist natural classes of sparse signals for which adaptivity would not improve the bounds on non-adaptivity. This is the case for the class of signals that has been considered in this simulations (i.e signal without any structure). However, one can think that for some specific class of signals, adaptivity (i.e. a judicious choice in the polar angles) could improve the performance on non-adaptivity (i.e. a random angle selection).

Limitations of the greedy algorithm - We point out the following two limitations in the proposed approach:

1. Algorithm 2 (as well as Algorithm 1 or Algorithm 1-bis) is not guaranteed to find the largest $\mathcal{D}_{x}$ for small values of $R$ and $Q$. For some given sparsity degree $s$, this may happen if $F$ is the $s$-dimensional face of the polytope that is the closest to the origin among all other $s$-faces. Now it might happen that some sub-faces of $F$, say $F^{\prime}$ of dimension $s^{\prime}<s$, are not the closest to the origin among all the $s^{\prime}$-dimensional faces of the polytope. If this happens, since the algorithm retains only vectors with increasing support, a suited minimal choice at one step $s^{\prime}$ may induce a wrong (non-minimal) choice at a later stage $s$. This is one tradeoff for the complexity gain.

However, the impact of this trade-off seems of little importance, as the algorithms clearly outperform a random search. In order to analyse this fact, we generate 100 vectors from a randomly generated support $I$ such that $|I|=s$ with $s=10, s=32$, and $s=50$. In Table 3, we present the maximum $\mathcal{D}_{x}$ value for vectors in $\Sigma_{\max }^{(s)}$ extracted with Algorithm 2 and for the random sparse vectors. We can clearly observe that for these tomography-like experiments, Algorithm 2 provides vectors with the largest $\mathcal{D}_{x}$ value. 


\begin{tabular}{|c|c|c|c|}
\hline & $\begin{array}{l}2 \text { views } \\
M=98 \\
\end{array}$ & $\begin{array}{c}4 \text { views } \\
M=196\end{array}$ & $\begin{array}{c}6 \text { views } \\
M=294\end{array}$ \\
\hline Uniform sampling & 10 & 32 & 50 \\
\hline Random sampling $n^{\circ} 1$ & 10 & 34 & 54 \\
\hline Random sampling $n^{\circ} 2$ & 14 & 25 & 47 \\
\hline Random sampling $\mathrm{n}^{\circ} 3$ & 8 & 26 & 60 \\
\hline Random sampling $\mathrm{n}^{\circ} 4$ & 9 & 27 & 58 \\
\hline Random sampling $n^{\circ} 5$ & 10 & 29 & 48 \\
\hline Random sampling $n^{\circ} 6$ & 7 & 18 & 50 \\
\hline Random sampling $n^{\circ} 7$ & 4 & 27 & 67 \\
\hline Random sampling $\mathrm{n}^{\circ} 8$ & 11 & 43 & 66 \\
\hline Random sampling $n^{\circ} 9$ & 11 & 23 & 40 \\
\hline Random sampling $n^{\circ} 10$ & 12 & 44 & 50 \\
\hline $\begin{array}{l}\text { Random sampling } \\
\text { (Minimum) }\end{array}$ & 4 & 18 & 40 \\
\hline $\begin{array}{l}\text { Random sampling } \\
\text { (Mean) }\end{array}$ & 9 & 29 & 54 \\
\hline $\begin{array}{l}\text { Random sampling } \\
\text { (Maximum) }\end{array}$ & 14 & 44 & 67 \\
\hline
\end{tabular}

Table 2: Sparsity extracted with Algorithm 4 for a uniform or a random sampling of the tomography matrix. 
2. It is not obvious that we have a uniform recovery for a given $\boldsymbol{A}$ using $\ell_{1}$-minimization. Indeed, whether a collection of vertices in the polytope forms a face that does not solely depend on how far their convex hull lies from the origin, one can easily define polytopes for which some faces are closer to the origin, while convex hulls of some other vertices lie farther but still remain inside the polytope.

Table 4 aims to illustrate the second limitation. This table summarizes the reconstruction error obtained by $\ell_{1}$-minimization for vectors in $\Sigma_{\max }^{(s)}$ (i.e. found by the algorithm) and for vectors characterized by a randomly generated support. We can remark that for $M=98$, even if Algorithm 2 allows to reach vectors with the largest $\mathcal{D}_{x}$ value, we can randomly generate non- $\ell_{1}$-identifiable vectors. This interesting result illustrates the fact that, for some particular shapes of the polytope $P$, the proposed method has to be improved.

Noisy measurements - In Table 5, we evaluate the maximal sparsity degree for exact recovery in the noisy case. For such a purpose we computed the upper bound of $\|\widehat{\boldsymbol{x}}-\overline{\boldsymbol{x}}\|^{2}$. This bound requires the knowledge of the support $I$, on which quantities like $\operatorname{ERC}(I)$ or $\left\|A_{I}^{*} \boldsymbol{n}\right\|_{2}$ depend. The tested support $I$ corresponds to the vector $\boldsymbol{x} \in \Sigma_{\max }^{(s)}$ with the largest $\mathcal{D}_{\boldsymbol{x}}$, extracted with Algorithm 2. For this support and for some fixed variance $\sigma$ of the additive noise, we generate 100 random realizations of a vector $\boldsymbol{n} \sim \mathcal{N}\left(0, \sigma^{2}\right)$. For each realization we compute the mean support-depending quantities involved in the upper bound and then estimate an approximated value of the error. This task is carried out for matrices of different size i.e. number of polar angle views, for supports $I$ of different sizes and for different values of the noise variance. The results are gathered in Table 5 . We can observe that the first row leads to the same sparsity degree that the one obtained in Table 1 when sparsity is extracted with ERC. Moreover, it can be observed that in the presence of noise (cf. rows 2 to 5 in Table 5) the sparsity degree decreases in order to achieve a smallest error.

\begin{tabular}{|c|c|c|c|}
\hline & $\begin{array}{l}2 \text { views } \\
M=98\end{array}$ & $\begin{array}{c}4 \text { views } \\
M=196\end{array}$ & $\begin{array}{c}6 \text { views } \\
M=294\end{array}$ \\
\hline $\begin{array}{l}\text { Maximum } \mathcal{D}_{x} \text { value } \\
\quad \text { for } \boldsymbol{x} \in \Sigma_{\max }^{(s)}\end{array}$ & $\begin{array}{c}(s=10) \\
\mathbf{3 . 1 5}\end{array}$ & $\begin{array}{c}(s=32) \\
\mathbf{3 . 1 4}\end{array}$ & $\begin{array}{c}(s=50) \\
\mathbf{2 . 5 2}\end{array}$ \\
\hline $\begin{array}{c}\text { Maximum } \mathcal{D}_{x} \\
\text { value for } \boldsymbol{x} \\
\text { having a randomly } \\
\text { generated support }\end{array}$ & $\begin{array}{c}(s=10) \\
\mathbf{2 . 8 1}\end{array}$ & $\begin{array}{c}(s=32) \\
\mathbf{2 . 6 2}\end{array}$ & $\begin{array}{c}(s=50) \\
\mathbf{2 . 2 5}\end{array}$ \\
\hline
\end{tabular}

Table 3: Maximum $\mathcal{D}_{x}$ value when $\boldsymbol{x}$ is extracted with Algorithm 2 or when $\boldsymbol{x}$ are generated randomly. 


\begin{tabular}{|c|c|c|c|}
\hline & $\begin{array}{c}2 \text { views } \\
M=98\end{array}$ & $\begin{array}{c}4 \text { views } \\
M=196\end{array}$ & $\begin{array}{c}6 \text { views } \\
M=294\end{array}$ \\
\hline \hline $\begin{array}{c}\text { Reconstruction error } \\
\text { for } \boldsymbol{x} \in \Sigma_{\max }^{(s)}\end{array}$ & $\begin{array}{c}(s=10) \\
<\mathbf{1 0}^{-\mathbf{3}}\end{array}$ & $\begin{array}{c}(s=32) \\
<\mathbf{1 0}^{-\mathbf{3}}\end{array}$ & $\begin{array}{c}(s=50) \\
<\mathbf{1 0}^{-\mathbf{3}}\end{array}$ \\
\hline \hline $\begin{array}{c}\text { Reconstruction error } \\
\text { for } \boldsymbol{x} \text { randomly } \\
\text { generated }\end{array}$ & $\begin{array}{c}(s=10) \\
<\mathbf{1}\end{array}$ & $\begin{array}{c}(s=32) \\
<\mathbf{1 0}^{-\mathbf{3}}\end{array}$ & $\begin{array}{c}(s=50) \\
<\mathbf{1 0}^{-\mathbf{3}}\end{array}$ \\
\hline
\end{tabular}

Table 4: Reconstruction error when $\boldsymbol{x}$ is extracted with Algorithm 2 or when $\boldsymbol{x}$ are generated randomly.

\begin{tabular}{|c|c|c|c|}
\hline & $\begin{array}{c}2 \text { views } \\
(M=98)\end{array}$ & $\begin{array}{c}4 \text { views } \\
(M=196)\end{array}$ & $\begin{array}{c}6 \text { views } \\
(M=294)\end{array}$ \\
\hline \hline $\begin{array}{c}\text { Error }=0 \\
\sigma^{2}=0\end{array}$ & 4 & 10 & 11 \\
\hline $\begin{array}{c}\text { Error } \leq 10^{-2} \\
\sigma^{2}=10^{-4}\end{array}$ & 4 & 10 & 11 \\
\hline $\begin{array}{c}\text { Error } \leq 10^{-3} \\
\sigma^{2}=10^{-4}\end{array}$ & 1 & 4 & 5 \\
\hline $\begin{array}{c}\text { Error } \leq 10^{-1} \\
\sigma^{2}=10^{-3}\end{array}$ & 4 & 10 & 11 \\
\hline $\begin{array}{c}\text { Error } \leq 10^{-2} \\
\sigma^{2}=10^{-3}\end{array}$ & 1 & 4 & 5 \\
\hline
\end{tabular}

Table 5: Sparsity $s$ allowing us to recover every $s$-sparse vectors by $\ell_{1}$-minimization with noise consideration. The results are obtained from Proposition 3.1 where $I$ denotes the support of the vector $x \in \Sigma_{\max }^{(s)}$ with the largest $\mathcal{D}_{\boldsymbol{x}}$. Results for three different configurations of the tomographylike matrix $\boldsymbol{A}$.

\section{Conclusion}

This work presents new theoretical results and algorithms for $\ell_{1}$-recovery conditions in a deterministic context in order to (i) improve the state-of-the-art methods in term of extracted sparsity degree and (ii) easily bound the reconstruction error. More specifically, we propose an efficient method to obtain an upper bound of the sparsity degree $s$ for which every $s$-sparse vector can be 
reconstructed by $\ell_{1}$-minimization according to a specific measurement matrix. An efficient greedy algorithm is proposed in order to deal with deterministic matrices. Moreover, the robustness to noise is studied and numerical experiments based on tomography-like sensing matrix illustrate the theoretical results.

We should notice that this approach can be considered for other contexts in inverse problems such as restoration or inpainting.

\section{References}

[1] Arias-Castro, E., Candès, E.J., Davenport, M.A.: On the Fundamental Limits of Adaptive Sensing. IEEE Trans. Inform. Theory 59(1), 472-481 (2013)

[2] Bach, F.R., Jenatton, R., Mairal, J., Obozinski, G.: Optimization with sparsity-inducing penalties. Foundations and Trends in Machine Learning 4(1), 1-106 (2012)

[3] Beck, A., Teboulle, M.: A fast iterative shrinkage-thresholding algorithm for linear inverse problems. SIAM J. Imaging Sci. 2(1), 183-202 (2009)

[4] Blumensath, T., Davies, M.E.: Iterative hard thresholding for compressed sensing. Appl. Comp. Harm. Analysis 27(3), 265-274 (2009)

[5] Bourgain, J., Dilworth, S.J., Ford, K., Konyagin, S., Kutzarova, D.: Explicit Constructions of RIP Matrices and Related Problems. Duke Mathematical Journal 159(1), 145-185 (2011)

[6] Candès, E.J., Fernandez-Granda, C.: Towards a mathematical theory of super-resolution. Comm. Pure Applied Math., To appear (2013).

[7] Candès, E.J. and Romberg, J.: Practical signal recovery from random projections. In: Wavelet Applications in Signal and Image Processing XI, Proc. SPIE, vol. 5914 (2004)

[8] Candès, E.J., Romberg, J., Tao, T.: Robust uncertainty principles: exact signal reconstruction from highly incomplete frequency information. IEEE Trans. Inform. Theory 52(2), 489-509 (2006)

[9] Combettes, P.L., Pesquet, J.C.: Proximal splitting methods in signal processing. In: H.H. Bauschke, R. Burachik, P.L. Combettes, V. Elser, D.R. Luke, H. Wolkowicz (eds.) Fixed-Point Algorithms for Inverse Problems in Science and Engineering, pp. 185-212. Springer-Verlag, New York (2010)

[10] Cormack, A.M.: Sampling the Radon transform with beams of finite width. Phys. Med. Biol. 23(6), 1141-1148 (1978)

[11] Davenport, M., Duarte, M.F., Eldar, Y.C., Kutyniok, G.: Introduction to compressed sensing. In: Compressed Sensing: Theory and Applications. Cambridge University Press (2011)

[12] DeVore, R.A.: Deterministic constructions of compressed sensing matrices. Journal of Complexity 23, 918-925 (2007) 
[13] Donoho, D.L.: Neighborly polytopes and sparse solutions of underdetermined linear equations. Tech. rep., Department of Statistics, Stanford University (2004)

[14] Donoho, D.L., Huo, X.: Uncertainty principles and ideal atomic decomposition. IEEE Trans. Inform. Theory 47(7), 2845-2862 (2001)

[15] Dossal, Ch., Mallat, S.: Sparse spike deconvolution with minimum scale. In: Proc. SPARS. Rennes, France (2005)

[16] Dossal, Ch., Peyré, G., Fadili, J.: A numerical exploration of compressed sampling recovery. Linear Algebra and Appl. 432(7), 1663-1679 (2010)

[17] Faridani, A.: Fan-bean tomography and sampling theory. Proc. of Symposia in Applied Mathematics 63, 43-66 (2006)

[18] Fuchs, J.: Recovery of exact sparse representations in the presence of bounded noise. IEEE Trans. Inform. Theory 51(10), 3601-3608 (2005)

[19] Fuchs, J.J.: On sparse representations in arbitrary redundant bases. IEEE Trans. Inform. Theory 50(6), 1341-1344 (2004)

[20] Grasmair, M., Haltmeier, M., Scherzer, O.: Necessary and sufficient conditions for linear convergence of $\ell_{1}$-regularization. Comm. Pure Applied Math. LXIV, 161-182 (2010)

[21] Gribonval, R., Nielsen, M.: Sparse representations in unions of bases. IEEE Trans. Inform. Theory 49(12), 3320-3325 (2003)

[22] Gribonval, R., Nielsen, M.: Highly sparse representations from dictionaries are unique and independent of the sparseness measure. Appl. Comp. Harm. Analysis 22(3), Elsevier - Academic Press (2007)

[23] Juditsky, A., Nemirovski, A.: On verifiable sufficient conditions for sparse signal recovery via $\ell_{1}$-minimisation. Math. Program., Ser. B 127, 57-88 (2011)

[24] Krzakala, F., Mézard, M., Sausset, F., Sun, Y.F., Zdeborová, L.: Statistical physics-based reconstruction in compressed sensing. Phys. Rev. X. 2(021005), x+18 (2012).

[25] Liang, D., Zhang, H.F., Ying, L.: Compressed-sensing photoacoustic imaging based on random optical illumination. Int. J. Functional Informatics and Personalised Medicine 2(4), 394-406 (2009)

[26] Lindgren, A.G., Rattey, P.A.: The inverse discrete Radon transform with applications to tomographic imaging using projection data. Advances in Electronics and Electron Physics 56, 359-410 (1981)

[27] Lu, Y., Zhang, X., Douraghy, A., Stout, D., Tian, J., Chan, T.F., Chatziioannou, A..F.: Source reconstruction for spectrally-resolved bioluminescence tomography with sparse a priori information. Optics Express 17(10), 8062-8080 (2009) 
[28] Pustelnik, N., Dossal, Ch., Turcu, F., Berthoumieu, Y., Ricoux, Ph.: A greedy algorithm to extract sparsity degree for $\ell_{1} / \ell_{0}$-equivalence in a deterministic context. In: Proc. Eur. Sig. and Image Proc. Conference, $\mathrm{x}+5$. Bucharest, Romania (2012)

[29] Rattey, P.A., Lindgren, A.G.: Sampling the 2-D Radon transforms. IEEE Trans. Acous., Speech Signal Process. 29, 994-1002 (1981)

[30] Seneviratne, A.J., Solo, V.: On vector $\ell_{1}$ penalized multivariate regression. In: Proc. Int. Conf. Acoust., Speech Signal Process., pp. x+4. Kyoto, Japan (2012)

[31] Starck, J.-L., Donoho, D., Fadili, M.J., Rassat, A.: Sparsity and the Bayesian Perspective. Astronomy and Astrophysics. (2013)

[32] Tropp, J.A.: Algorithms for simultaneous sparse approximation. Part II: Convex relaxation. Signal Process. 86, 589-602 (2006)

[33] Tropp, J.A.: Just relax: Convex programming methods for identifying sparse signals in noise. IEEE Trans. Inform. Theory 52, 1030-1051 (2006) 